\title{
The Anatomical Dry Eye
}

\section{-A Different Form of Ocular Surface Disease Deserves Focus}

\section{Gysbert van Setten*}

St Eriks Eye Hospital, Karolinska Institutet, Department of Clinical Neuroscience, Polhemsgatan 50, 11282 Stockholm, Sweden

Email: gysbert-botho.vansetten@sll.se

How to cite this paper: van Setten, G. (2017) The Anatomical Dry Eye-A Different Form of Ocular Surface Disease Deserves Focus. Open Journal of Ophthalmology, 7, 184-190.

https://doi.org/10.4236/ojoph.2017.73025

Received: May 2, 2017

Accepted: July 14, 2017

Published: July 17, 2017

Copyright $\odot 2017$ by author and Scientific Research Publishing Inc. This work is licensed under the Creative Commons Attribution International License (CC BY 4.0).

http://creativecommons.org/licenses/by/4.0/

\begin{abstract}
Introduction: Dry eye disease is currently considered mainly tear film related ocular surface condition. This concept does, however, not respect ocular surface topography. The micro-anatomy of the corneal changes may lead to enhanced demands on the tear film and lead to significant complaints. However, they often remain undetected and hence untreated. It is suggested that the pathophysiology for an entire subgroup of dry eye disease patients is primarily of surface morphological nature. Methods. The tear film break up was observed and used to identify anatomical alterations in eyes of patients with dry eye complaints. The localization and pattern of TFBUT using fluorescein was compared between eye with normal surfaces and surface alterations. Results. Premature tear film rupture was localized at constantly same areas and did match changes on the ocular epitheliopathy in patients with diseases such as microcystic epithliopathy, MFD, and after excimer laser treatment. Disusssion: Whereas in normal surfaces TFBUT does occur within the floating tear film, the anatomical dry eye identifies itself with constant location of tear film break up and a constant spreading pattern. In contrast to the classic, tear film caused dry eye, the anatomical dry eye is accessible to treatment. This should catch our attention and intent to identify it. It is the ease of possible treatment that should make these ocular surface alterations prime target of dry eye disease diagnostic. It is hence suggested to introduce the anatomical dry eye as a subgroup in the large group of dry eye and ocular surface disease.
\end{abstract}

\section{Keywords}

Dry Eye, Ocular Surface, Break Up TIME, BUT, Anatomy, Surgery

\section{Introduction}

The multicausal pathophysiology behind the phenomen which we understand as dry eye disease is commonly accepted. In the current specification of dry eye ${ }^{*} \mathrm{MD}, \mathrm{PhD}$. 
disease main attention has been given to insufficiencies of the lacrymal system and the medium covering the surface of the eye, the tear film. Sophisticated methods to identify and to grade the dryness and its effects at the surface have been developed. The term dry eye itself is associated with the change of tear film parameters and corresponding surface alterations. One of them, the tear film break up time (TFBUT) (Mengher et al. 1985) [1], is a parameter reflecting tear film integrity and function, measuring the time it takes that the tear film ruptures after opening the eye. Measured in seconds, the TFBUT should be in the healthy eye more than 10 seconds (Lemp and Hamill 1973) [2] or at least $5 \mathrm{sec}$ (Abelson et al. 2002) [3]. The TFBIT is measured after application of fluorescein and observing the first sign of the disrupture of the homogenous greenish tear film layer, visualized by the appearance of black, rapidly enlarging, fluorescein free areas. It is known that various conditions such as allergy may cause a shorter TFBUT (Fujishima 1996) [4]. However, commonly, the surface is considered as a whole, uniform unit and judged as such. To perceive dry eye uncritically, this way does, however, deprive us from the basic opportunity to differ between different anatomical forms of dry eye. Focused on the tear film and its composition and effects on vision, the anatomical pathophysiology for dry eye at the surface has so far not received major attention. The anatomical dry eye has this far not been identified as a subgroup of dry eye disease. After 30 years of investigating, the staining pattern of ocular surfaces in different forms of ocular disease and specific forms of dry eye were identified. That deserves to be recognized and does differ from the classic form of dry eye, where the tear film deficiencies are the main reason for the surface disease. The key target of the present approach was to identify a clear and easy method to distinguish the classic form of dry eye from the anatomical form, the secondary form of dry eye.

\section{Materials and Methods}

Patients with normal surfaces and patients with corneal scars, laser treated corneas, eyes with microcystic epitheliopathy, Map and finger print dystrophy (MFD) were investigated. As key tool for differentiating diagnosis was simple fluorescein staining used. Slitlamp photography was conducted using a cobalt blue filter and a yellow filter. Using this setting the tear film was observed after application of fluorescein. After three to four blinks the patients were instructed to cease blinking and to keep the eyes open. The classical tear film break up was identified by the visibility of the first interruption of the homogenous yellowish tear film layer. The location and spreading pattern of this site was observed and followed over 30 seconds. Attention was paid to the localization of the break up site, i.e. whether it did slightly move within the tear film layer or whether it was fixed to a certain location and did not move at all in its position at the ocular surface.

\section{Results}

The anatomical surface morphology causes localized thinning of the tear film. This rupture of the film does present itself to the uncritical observer in the same 
way as the common break up phenomen. However, it is characterized by two characteristic features: 1) It's localization which remains the same during the entire time of observation and is repeatedly visible at the same location, independent how often staining is performed and 2) The constant form of break up process. In contrast to the varying pattern of tear fluid break on a surface covered with a homgenously evenly thick tear film, the anatomical dry eye has the same appearance and shape of the break up region every time staining is performed. (Figure 1). These two characteristics will identify the presence of an anatomical dry eye or a premature break up of the tear film. Additional features such as the specific shape of contour-anomaly caused by a corneal flap after excimer laser treatment, Map and Finger Print Dystrophy and others (Figure 2) may further help to identify the anatomical dry eye and differentiate it from the classic, solely tear film related break up time (BUT). A microcyst does constitute a local elevation that, with decreasing tear film thickness during the opening of the eye, will lead to premature tear film break up just at this exact location (Figure 2(a)).

Similarly do local micro-topographical surface alterations such as epithelial changes lead to enhanced demands on the tear film that will lead to localized rupture of the tear film layer (Figure 2(b)), of which MFD is a very easily identifiable feature (Figure 2(c)). Similarly do other microscopic changes in the surface homogeneity such as local epithelial hyperplasia (Figure 2(d), arrow) lead to local tear film functional insufficiency, as do local single cell changes (Figure $2(\mathrm{e})$ ). The laser treated eye often has a permanently visible edge of where the border of the flap has reattached (Figure 2(f)). It is in this area where abnormal tear film break up may occur.

\section{Discussion}

Most of these surface alterations reveal themselves only to the prudent observer and under high magnification. The anatomical dry eye has specific features that do allow to identify its presence: constant location of tear film break up and its constant appearance (spreading pattern). The anatomical nature of the causing pathology allows in many cases treatment. This is especially true for

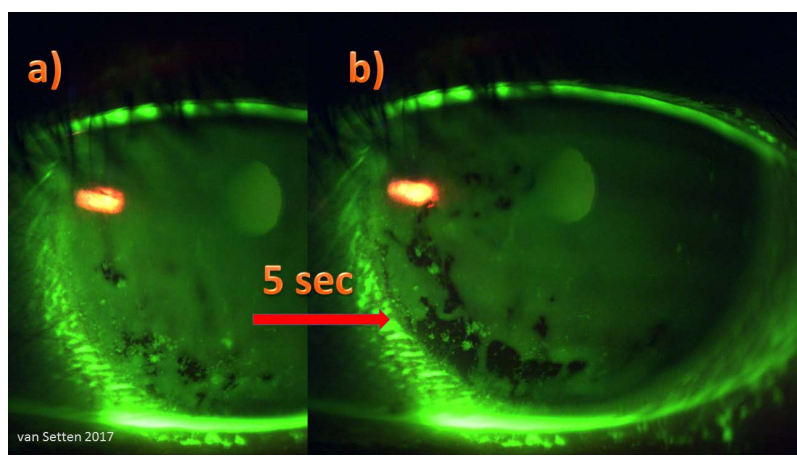

Figure 1. Typical appearance of the anatomical dry eye: Localized premature (early) break up of the fluorescein layer in tear film over a region with minimal topographical anomalities. 

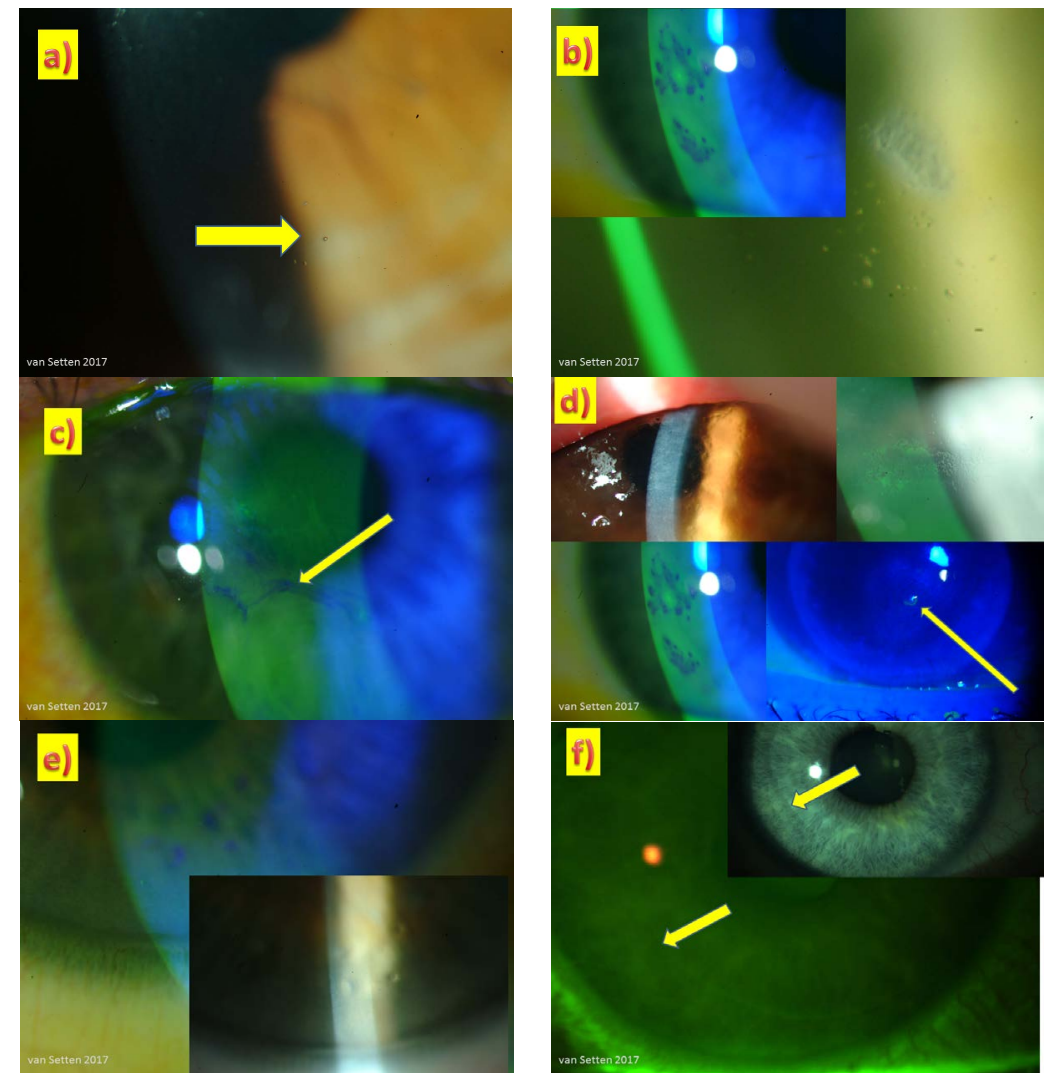

Figure 2. Premature tear film break up at a location of a microcyst (1a). Local micro-topographical surface alterations such as epithelial changes lead to localized rupture of the tear film layer (b). Localized fluorescein break up in an eye with MFD which is a very easily identifiable feature (c). Similarly do other microscopic changes in the surface homogeneity such as local epithelial hyperplasia ((d), arrow) lead to local tear film functional insufficiency, also do local single cell changes lead to premature tear film rupture (e). The laser treated eye not seldom has a permanently visible edge of where the border of the flap has reattached (f). It is in this area where abnormal tear film break up may occur.

strictly epithelial alterations such a s microcysts and corneal dystrophies (Germundsson et al., 2010) [5] which are rather common (Werblin et al. 1981) [6]. Here especially map and fingerprint dystrophies have been shown to be very accessible to excimer laser (Örndahl et al. 1998) [7]. Other surface alterations have been already early become very early targeted by the option of excimer laser treatment (Förster et al. 1997) [8].

From the vast pool of ocular surface disease here only a few diseases were identified in this study. Conditions that simulate ocular surface dryness for the uncritical observer. Of course there is not always a clear distinction and mixed forms of anatomical and primary tear film related dry eye disease may exist. This the more as with increasing age tear film parameters may change (Mathers et al. 1996) [9] and subclinical conditions of a decreasing tear film composition and function may pair with a potentially age related increase of the clinical significance of corneal dystrophies. Regardless of this, however, this study points out that BUT as a sign of qualitative tear film insufficiency, i.e. enhanced instability can only be used as reliable parameter if the ocular surface does not show any 
anatomical anomaly that results in changes in surface morphology. This is very important as surface inhomogeneity causing premature BUT may be very subtle, such as singular microcysts (Figure 2) or minor deposits.

Due to pathophysiology and the treatment options it is suggested to differ between a dry eye caused by anatomical inhomogeneity of the ocular surface profile and the forms of dry eye where the surfaces are even, homogenous in their surface profile, the classic dry eye. The real, classic tear film breakup does occur within the film. Therefore, the site of TF BU is slightly moving on/in the film of tears and changing its location. The premature, anatomical tear film break up occurs at local areas of tear film thinning caused by the irregularities of the surface under the film, which, by their nature, are adherent to their location. In eyes without anatomical alterations of the ocular surface, a tendency of tear breakup to preferentially close to the central corneal region was observed. This is in line with the observations of Liu et al. (2006) [10].

The anatomical dry eye does, however, focus on the conditions of the corneal surface itself and its effect on break up time of the tear film. It does not consider the pathophysiology of the lids such as described by Taylor outlining discontinuity of the marginal tear strip in association with early break up time due to conjunctival scarformation (Taylor 1980) [11]. Nevertheless, it is here that corneas micro-anatomy does correlate to perceived dry eye complaint after cataract (Cho and Kim 2009) [12] or laser surgery (Ang et al. 2001 [13], Toda et al. 2001 [14]). It remains to be clarified, if it is justified to consider excimer laser surgery related dry eye complaints as a neuropathic disease (Wilson 2001 [15], Chao et al. 2015 [16]) although evidence for anatomical reasons are present. Possibly the algorithm and technique (Huang et al. 2012) [17] decide on the pathophysiology of dry eye development after laser surgery. It is on the other hand the excimer laser which used on phototherapeutic keratectomy (PTK) may be used to even out some of the epithelial contour anomalies causing dry eye complaints (Germundson et al. 2011).

\section{Conclusion}

The anatomical dry eye has specific features that do allow identifying its presence: constant location of tear film break up and its constant appearance (spreading pattern). It may occur even if the tear film as medium covering the surface is in its composition and quality within normal limits. However, surface irregularities or topographical alterations may exceed the physical-chemical performance-abilities of the tears and lead to a rupture of tear film ahead of the normal tear film break up time. As the reason of this phenomenon is not found within the tears but is (micro-) anatomical by its nature, it is suggested to consider this group as a group of its own within those with dry eye diseases as defined by DEWS [18], i.e. the anatomical dry eye.

\section{Acknowledgements}

The author is very thankful to Aviation-Ophthalmology, Stockholm, Sweden for 
all technical support.

\section{References}

[1] Mengher, L.S., Bron, A.J., Tonge, S.R. and Gilbert, D.J. (1985) A Non-Invasive Instrument for Clinical Assessment of the Pre-Corneal Tear Film Stability. Current Eye Research, 4, 1-7.

[2] Abelson, M.B., Ousler III, G.W., Nally, L.A., Welch, D. and Krenzer, K. (2002) Alternative Reference Values for Tear Film Break Up Time in Normal and Dry Eye Population. Advances in Experimental Medicine and Biology, 506, 1121-1125. https://doi.org/10.1007/978-1-4615-0717-8_55

[3] Lemp, M.A. and Hamill, J.R. (1973) Factors Affecting Tear Film Breakup in Normal Eyes. Arch Ophthalmology, 89, 103-105. https://doi.org/10.1001/archopht.1973.01000040105007

[4] Fujishima, H., Toda, I., Shimazaki, J. and Tsubota, K. (1996) Allergic Conjunctivitis and Dry Eye. British Journal of Ophthalmology, 80, 994-997. https://doi.org/10.1136/bjo.80.11.994

[5] Germundsson, J., Fagerholm, P. and Lagali, N. (2011) Clinical Outcome and Recurrence of Epithelial Basement Membrane Dystrophy after Phototherapeutic Keratectomy. Ophthalmology, 118, 515-522. https://doi.org/10.1016/j.ophtha.2010.07.003

[6] Werblin, T.P., Hirst, L.W., Stark, W.J. and Maumenee, I.H. (1981) Prevalence of Map-Dot-Fingerprint Changes in the Cornea. British Journal of Ophthalmology, 65, 401-409. https://doi.org/10.1136/bjo.65.6.401

[7] Örndahl, M.J.F. and Fagerholm, P.P. (1998) Phototherapeutic Keratectomy for Map-Dot-Fingerprint Corneal Dystrophy. Cornea, 17, 595-599. https://doi.org/10.1097/00003226-199811000-00004

[8] Förster, W., Atzler, U., Ratkay, I. and Busse, H. (1997) Therapeutic Use of the 193nm Excimer Laser in Corneal Pathologies. Graefe's Archive for Clinical and Experimental Ophthalmolog, 235, 296-305. https://doi.org/10.1007/BF01739639

[9] Mathers, W.D., Lane, J.A. and Zimmerman, M.B. (1996) Tear Film Changes Associated with Normal Aging. Cornea, 15, 229-234. https://doi.org/10.1097/00003226-199605000-00001

[10] Liu, H., Begley, C.G., Chalmers, R., Wilson, G., Srinivas, S.P. and Wilkinson, J.A. (2006) Temporal Progression and Spatial Repeatability of Tear Breakup. Optometry and Vision Science, 83, 723-730. https://doi.org/10.1097/01.opx.0000237546.88464.6d

[11] Taylor, H. (1980) Studies on the Tear Film in Climatic Droplet Keratopathy and Pterygium. Archives of Ophthalmology, 98, 86-88. https://doi.org/10.1001/archopht.1980.01020030088005

[12] Cho, Y.K. and Kim, M.S. (2009) Dry Eye after Cataract Surgery and Associated Intraoperative Risk Factors. Korean Journal of Ophthalmology, 23, 65-73. https://doi.org/10.3341/kjo.2009.23.2.65

[13] Ang, R.T., Dartt, D.A. and Tsubota, K. (2001) Dry Eye after Refractive Surgery. Current Opinion in Ophthalmology, 12, 318-322. https://doi.org/10.1097/00055735-200108000-00013

[14] Toda, I., Asano-Kato, N., Komai-Hori, Y. and Tsubota, K. (2001) Dry Eye after Laser in Situ Keratomileusis. American Journal of Ophthalmology, 132, 1-7. https://doi.org/10.1016/S0002-9394(01)00959-X

[15] Wilson, S.E. (2001) Laser in Situ Keratomileusis-Induced (Presumed) Neurotrophic 
Epitheliopathy. Opthalmology, 108, 1082-1087.

https://doi.org/10.1016/S0161-6420(01)00587-5

[16] Chao, C., Stapleton, F., Zhou, X., Chen, S., Zhou, S. and Golebiowski, B. (2015) Structural and Functional Changes in Corneal Innervation after Laser in Situ Keratomileusis and Their Relationship with Dry Eye. Graefe's Archive for Clinical and Experimental Ophthalmology, 253, 2029-2039.

https://doi.org/10.1007/s00417-015-3120-1

[17] Huang, J.C., Sun, C.C., Chang, C.K., Ma, D.H. and Lin, Y.F. (2012) Effect of Hinge Position on Corneal Sensation and Dry Eye Parameters after Femtosecond LaserAssisted LASIK. Journal of Cataract \& Refractive Surgery, 28, 625-631. https://doi.org/10.3928/1081597X-20120815-07

[18] Listed, N. (2007) Research in Dry Eye. Report of the Research Subcommittee of the International Dry Eye Work Shop. The Ocular Surface, 5, 179-193.

https://doi.org/10.1016/S1542-0124(12)70086-1

Submit or recommend next manuscript to SCIRP and we will provide best service for you:

Accepting pre-submission inquiries through Email, Facebook, LinkedIn, Twitter, etc. A wide selection of journals (inclusive of 9 subjects, more than 200 journals)

Providing 24-hour high-quality service

User-friendly online submission system

Fair and swift peer-review system

Efficient typesetting and proofreading procedure

Display of the result of downloads and visits, as well as the number of cited articles

Maximum dissemination of your research work

Submit your manuscript at: http://papersubmission.scirp.org/

Or contact ojoph@scirp.org 